\title{
Morbidade neonatal dos recém-nascidos pré-termo tardios comparados aos de termo precoce
}

\author{
Neonatal morbidity of late preterm compared with early term neonates
}

Nuno Lourenço ${ }^{1} \bowtie$, Marco Fernandes ${ }^{1}$, Clara Gomes ${ }^{1}$, Cristina Resende ${ }^{1}$

${ }^{1}$ Maternidade Bissaya Barreto, Centro Hospitalar e Universitário de Coimbra. Coimbra, Portugal.

\section{RESUMO}

Objetivos: Avaliar e comparar a morbidade neonatal entre recém-nascidos pré-termo tardios (RNPT-T) e recém-nascidos de termo precoce (RNT-P).

Métodos: Estudo transversal retrospetivo, incluindo nascidos vivos em uma maternidade hospitalar de referência regional em Portugal, nos anos de 2014 e 2015, com idade gestacional entre $34^{0 / 7}$ e $38^{6 / 7}$ semanas. Os RNPT-T (34/7-36 $6{ }^{6 / 7}$ semanas) foram considerados casos e os RNT-P (37/7-38 nhamento pré-natal e internação por motivo social ou causa materna. Foi avaliada e comparada a morbidade neonatal entre os dois grupos, utilizando o teste $t$ de Student para comparação entre médias (variáveis contínuas) e o Qui-quadrado ou teste de Fisher para comparação entre variáveis categóricas. As odds ratio (OR) com seus respetivos intervalos de confiança (IC) $95 \%$ foram ajustadas (ORa) ao peso de nascimento, tipo de parto, patologia materna e gemelaridade. Considerou-se o valor $\mathrm{p}<0.05$ como estatisticamente significativo.

Resultados: Foram avaliados 1.745 recém-nascidos, sendo 324 casos (RNPT-T) e 1.421 controles (RNT-P). Os RNPT-T associaram-se a maior frequência de hipertensão materna e diabetes gestacional e maiores taxas de gemelaridade e de cesariana. Os RNPT-T tiveram também um maior risco de necessidade de reanimação $(\mathrm{OR}=2,0 \mathrm{IC} 95 \%$ 1,3-3,0); hipoglicemia $(\mathrm{ORa}=4,9 \mathrm{IC} 95 \%$ 2,9-8,2); hiperbilirrubinemia $(\mathrm{ORa}=4,8$ IC95\% 3,7-6,2), taquipneia transitória (ORa=6,4 IC95\% 4,0-10,3); dificuldades alimentares (ORa=6,6 IC95\% 4,8-8,9); sepsis $(\mathrm{ORa}=4,4 \mathrm{IC} 95 \%$ $4,8-8,9)$; permanência hospitalar $\geq 5$ dias (ORa=8,6 IC95\% 6,6-11,3); e menor taxa de aleitamento materno exclusivo (ORa=0,2 IC95\% 0,15-0,3). Conclusões: Em comparação aos RNT-P, os RNPT-T apresentaram maior risco de morbidade. Este estudo reforça a necessidade de um seguimento clínico apropriado aos RNPT-T no período neonatal.

DESCRITORES: recém-nascido; prematuro; morbidade.

\section{ABSTRACT}

Aims: To evaluate and compare neonatal morbidity between late-preterm infants (LPTI) and early term infants (ETI).

Methods: Retrospective cross-sectional study, including live births at a regional referral maternity hospital in Portugal, in the years 2014 and 2015, with gestational age between $34^{0 / 7}$ and $38^{6 / 7}$ weeks. LPTI $\left(34^{0 / 7}-36^{6 / 7}\right.$ weeks) were considered as cases and ETI (370/7 $-38^{6 / 7}$ weeks) were considered controls. Neonates with congenital malformations, lack of prenatal care and hospitalization for social/maternal reasons were excluded. We evaluated and compared neonatal morbidity between the two groups, using Student's t-test for comparison between means (continuous variables) and Chi-square or Fisher's test for comparison between categorical variables. Odds ratio (OR) was calculated and adjusted (aOR) to birth weight, maternal disease, type of birth and multiple pregnancy. A value of $\mathrm{p}<0,05$ was considered of statistical significance.

Results: A total of 1,745 neonates were evaluated: 324 cases (LPTI) and 1,421 controls (ETI). LPTI was associated with a higher frequency of maternal hypertension and gestational diabetes and higher rates of twinning and cesarean section. LPTI also had a higher risk for resuscitation $(\mathrm{OR}=2.0-95 \% \mathrm{CI} 1.3-3.0)$; hypoglycemia $(\mathrm{aOR}=4.9-95 \% \mathrm{CI} 2.9-8.2)$; hyperbilirubinemia $(\mathrm{aOR}=4.8-95 \% \mathrm{CI} 3.7-6.2)$, transient tachypnea $(\mathrm{aOR}=6.4-95 \% \mathrm{CI} 4.0-10.3)$; eating difficulties $(\mathrm{aOR}=6.6-95 \% \mathrm{CI} 4.8-8.9)$; sepsis $(\mathrm{aOR}=4.4-95 \% \mathrm{CI} 4.8-8.9)$; hospital stay $\geq 5$ days $(\mathrm{aOR}=8.6-95 \% \mathrm{CI} 6.6-11.3)$; and lower exclusive breastfeeding rate $(\mathrm{aOR}=0.2-95 \% \mathrm{CI} 0.15-0.3)$.

Conclusions: In comparison to ETI, LPTI presented a higher risk of morbidity. This study reinforces the need for appropriate clinical followup of LPTI in the neonatal period.

KEY WORDS: infant, newborn; infant, premature; morbidity. 
Abreviaturas: DG, diabetes gestacional; EHI, encefalopatia hipóxico-isquêmica; GIG, grande para a idade gestacional; HPIV, hemorragia peri/intraventricular ; IG, idade gestacional; IC, intervalos de confiança ; PIG, pequeno para a idade gestacional; OR, odds ratio; RNT-P, recém-nascidos de termo precoce; RNPT-T, recém-nascidos pré-termo tardios; TTRN, taquipneia transitória do recém-nascido; UCIN, unidade de cuidados intensivos.

\section{INTRODUÇÃO}

Segundo a Organização Mundial da Saúde (OMS), um parto considerado a termo é o que ocorre entre 37 e 42 semanas de idade gestacional [1]. Esta definição data da década de 1970 e, já nessa época, alguns perinatologistas preferiam adiar a definição de termo para as 38 semanas, pois consideravam que antes dessa idade gestacional o recém-nascido ainda seria imaturo. Contudo, a definição de prematuridade da OMS já estava enraizada, pelo que se manteve as 37 semanas como limite inferior da definição de parto a termo.

O aumento do número de recém-nascidos prétermo assume-se como um problema não só pelo seu aumento, mas pelos cuidados de saúde que acarretam. Nos Estados Unidos da América, por exemplo, houve um aumento de $25 \%$ na taxa de nascimentos prematuros entre 1990 e 2005, com os partos pré-termo perfazendo cerca de $12,5 \%$ de todos os partos. Dentro destes, cerca de $70 \%$ correspondiam a partos pré-termo tardios, ou seja, entre as $34^{0 / 7}-36^{6 / 7}$ semanas de gestação [2]. Em Portugal, um estudo sobre a prevalência de partos pré-termo tardios e termo precoces (partos ocorridos entre as $37^{0 / 7}$ e $38^{6 / 7}$ semanas de gestação) estimou uma prevalência de $5,4 \%$ de partos pré-termo tardios e $27 \%$ de termo precoces em 2013 [3].

As evidências cada vez maiores da imaturidade fisiológica e metabólica dos recém-nascidos pré-termo tardios (RNPT-T) levou à alteração da definição de "near term" ("quase termo"), para "late preterm" ("pré-termo tardio"), por parte do National Institute of Child Health and Human Development (NICHHD), realçando as diferenças dos RNPT-T em relação aos de termo $[2,4]$. Essas diferenças entre morbidades neonatais ocorrem mesmo entre os recém-nascidos de termo, pelo que o American College of Obstetricians and Gynecologists e a Society for Maternal-Fetal Medicine propõem a classificação de recém-nascido de termo precoce (RNT-P), os "early term", para os nascidos entre as $37^{0 / 7} \mathrm{e}$ as $38^{6 / 7}$ semanas de gestação [5]. Isto porque essa idade gestacional está associada à maior morbidade neonatal em comparação aos recém-nascidos cujos partos ocorreram a partir das 39 semanas completas de gestação [6-8].

Mesmo reconhecendo a elevada morbidade dos RNT-P em relação aos recém-nascidos mais maduros, verifica-se nos RNPT-T ainda maior incidência de complicações respiratórias, instabilidade térmica, dificuldades alimentares, hipoglicemia neonatal, hiperbilirrubinemia, hemorragia peri-intraventricular (HPIV) e infecções [2,9,10]. Além disso, está descrito maior risco de morbidade a longo prazo nessas crianças, como atraso do desenvolvimento psicomotor e dificuldades de aprendizagem [11].

Com o aumento de nascimentos de RNPT-T e RNT-P, por vários motivos, como existência de novos métodos de fertilização e consequente aumento no número de gestações gemelares; crescente número de gestações no grupo de mulheres com mais de 35 anos; e aumento das indicações médicas para partos mais precoces, inclusive por cesarianas, devido ao acompanhamento pré-natal mais rigoroso, torna-se cada vez mais imperativo conhecer as possíveis morbidades desses recém-nascidos e o seu impacto no período neonatal $[2,4]$.

O objetivo deste trabalho foi avaliar e comparar a morbidade neonatal entre RNPT-T (grupo de casos) e RNT-P (grupo controle).

\section{MÉTODOS}

Um estudo transversal retrospetivo incluiu recémnascidos com idade gestacional de $34^{0 / 7}-38^{6 / 7}$ semanas, cujos partos ocorreram durante um período de dois anos (de 01/01/2014 a 31/12/2015) na Maternidade Bissaya Barreto, que pertence ao Centro Hospitalar e Universitário de Coimbra, Portugal, o qual possui apoio perinatal diferenciado e é referência de nível regional para situações de alto risco obstétrico/ neonatal, contando com uma Unidade de Cuidados Intensivos Neonatais (UCIN).

Os pacientes foram divididos em grupos de casos e de controles. Os casos foram definidos como os RNPT-T (34/7-36 6 semanas) e os controles definidos como os RNT-P (370/7-386/7 semanas). Foram excluídos recém-nascidos com malformações congênitas, ausência de cuidado pré-natal, hospitalizações prolongadas por motivo social ou doença materna, e patologias específicas, como por exemplo doenças neuromusculares e isoimunização Rh.

As variáveis neonatais analisadas foram sexo, peso de nascimento, necessidade de internação em UCIN, necessidade de reanimação no parto, taquipneia transitória do recém-nascido (TTRN), 
apneias da prematuridade, necessidade de ventilação assistida, necessidade de oxigênio suplementar, hiperbilirrubinemia (bilirrubina total sérica com indicação para realizar fototerapia de acordo com o protocolo da instituição), hipoglicemia (glicemia $<40 \mathrm{mg} / \mathrm{dl}$ ), sepsis neonatal, duração da internação (em dias), dificuldades alimentares, perda ponderal $>10 \%$, hemorragia peri e intraventricular (HPIV), lesão do plexo braquial, alta com aleitamento materno (LM) exclusivo, reinternação, encefalopatia hipóxicoisquêmica (EHI) e síndrome de aspiração meconial.

As variáveis maternas ou relacionadas com a gestação analisadas foram idade materna (em anos), paridade, tipo de parto (vaginal ou cesariana), gemelaridade, diabetes gestacional (DG), síndromes hipertensivas como (pré-eclâmpsia, hipertensão materna), indução ou não do trabalho de parto.

Sepsis neonatal foi definida como apresentação de clínica sugestiva associada a parâmetros de infeção positivos (proteína $\mathrm{C}$ reativa $>2 \mathrm{mg} / \mathrm{dl}$ e/ou leucócitos $>30.000 / \mu 1$ ou $<5.000 / \mu 1$ ), com ou sem isolamento de bactéria de acordo com os critérios definidos pelo Programa Nacional de Controlo de Infeções de Portugal [12].

A presença de EHI no recém-nascido foi definida na presença de alteração do estado de consciência, tônus, reflexos ou autonomia respiratória, associada a um índice de Apgar $\leq 5$ aos 5 e aos 10 minutos de vida, $\mathrm{pH}$ arterial $<7,2$ e déficit de bases $\geq 12 \mathrm{mmol} / \mathrm{L}$ nos primeiros 60 minutos de vida, presença de lesões na ressonância magnética sugestivas de EHI e falência multiorgânica [13].

O protocolo da instituição para realização de fototerapia nos prematuros é baseado no estudo de Okumura et al. [14]. Nos recém-nascidos de termo, o protocolo utilizado é baseado nas diretrizes do National Collaborating Centre for Women's and Children's Health [15].

Foi realizada ecografia transfontanelar em todos os recém-nascidos internados na UCIN. A HPIV foi classificada de acordo com os critérios de Volpe [16]. Definimos como recém-nascido pequeno para a idade gestacional (PIG) quando o peso de nascimento foi inferior ao percentil 3 para a idade gestacional, e grande para a idade gestacional (GIG) se o peso foi superior ao percentil 97 nas curvas de Fenton [17].

A análise estatística foi efetuada por meio do programa IBM SPSS, versão 20.0. Foi feita a análise bivariada usando o teste $t$ de Student para comparação entre médias (variáveis contínuas) e o teste Quiquadrado ou teste de Fisher para comparação entre variáveis categóricas. Calcularam-se as odds ratio
(OR) e os respetivos intervalos de confiança (IC) a $95 \%$. Foi considerada significância estatística se $\mathrm{p}<0,05$. As $\mathrm{OR}$ foram ajustadas para tipo de parto, gemelaridade, patologia materna e peso de nascimento.

O projeto deste estudo foi previamente aprovado pela Comissão de Ética da instituição em que a investigação foi realizada, e o protocolo seguiu todas os preceitos éticos da Declaração de Helsinque da Associação Médica Mundial e do International Committee of Medical Journal Editors.

\section{RESULTADOS}

Durante o período do estudo houve 336 partos entre as $34^{0 / 7}$ e as $36^{6 / 7}$ semanas de gestação e 1.461 partos entre as $37^{0 / 7}$ e as $38^{6 / 7}$ semanas de gestação. Após exclusão de acordo com os critérios referidos na metodologia, foram selecionados 324 RNPT-T (casos) e 1.421 RNT-P (controles), somando uma amostra total de 1.745 recém-nascidos. A distribuição por idade gestacional foi: 34 semanas de gestação $60(3,4 \%)$; 35 semanas $102(5,8 \%) ; 36$ semanas $162(9,3 \%) ; 37$ semanas 400 (22,9\%); e 38 semanas 1021 (58,5\%). O parto foi induzido em $30 \%$ nos dois grupos.

A Tabela 1 compara os dois grupos em relação a características dos recém-nascidos e fatores relacionados com a gestação e com o parto. Comparando os RNPT-T com os RNT-P não houve diferenças significativas de sexo, proporção de mães com mais de 35 anos ou proporção de mães primíparas.

Os RNPT-T associaram-se de forma significativa à maior frequência de patologia materna, como diabetes, hipertensão arterial e pré-eclâmpsia. Entre os RNPT-T houve uma percentagem de gêmeos 11 vezes superior. Em relação ao peso de nascimento, houve maior taxa de recém-nascidos tanto PIG quanto GIG nos RNPT-T, assim como um risco duas vezes maior de necessidade de reanimação na sala de parto (Tabela 1).

A Tabela 2 compara os dois grupos em relação à morbidade e mortalidade neonatais. Os RNPT-T quando comparados com os RNT-P (mesmo após a ajuste para patologia materna, peso nascimento e gemelaridade), foram associados a maior necessidade de internação em UCIN, incidência de TTRN, apneias, hiperbilirrubinemia, hipoglicemia, dificuldades alimentares, alta com leite adaptado, HPIV e hospitalização mais prolongada. Em termos de mortalidade houve apenas um óbito nos casos e outro nos controles, portanto sem significância estatística.

A morbidade neonatal variou inversamente com a idade gestacional: quanto menor a idade gestacional maior o risco de morbidade neonatal dos RNPT-T em 
relação aos RNT-P. A alta com aleitamento materno exclusivo variou diretamente em relação à idade gestacional. Os recém-nascidos com menor idade gestacional tiveram menos chance de alta com leite materno exclusivo, principalmente aqueles com menos de 36 semanas (Tabela 3 e Figura 1).

Tabela 1. Comparação entre os recém-nascidos pré-termo tardios (RNPT-T) e os recém-nascidos de termo precoce (RNT-P) em relação a fatores da gestação e do parto. Amostra total: 1745 recém-nascidos. Maternidade Bissaya Barreto, Centro Hospitalar e Universitário de Coimbra, Portugal, 2014/2015.

\begin{tabular}{|c|c|c|c|c|}
\hline Características & $\begin{array}{c}\text { RN } 34^{0 / 7}-36^{6 / 7} \\
(n=324)\end{array}$ & $\begin{array}{c}\text { RN } 37^{0 / 7}-38^{6 / 7} \\
(n=421)\end{array}$ & $\mathbf{p}^{*}$ & OR $(95 \%$ IC) \\
\hline Sexo & $180(56 \%)$ & 768 (54\%) & 0,62 & \\
\hline Idade materna $\geq 35$ anos & $113(34,9 \%)$ & $511(36,0 \%)$ & 0,89 & \\
\hline Mãe prímipara & $160(49,4 \%)$ & $685(48,2 \%)$ & 0,72 & \\
\hline Diabetes gestacional & $34(10,5 \%)$ & $78(5,5 \%)$ & 0,001 & $2,0(1,3-3,1)$ \\
\hline Hipertensão materna & $50(15 \%)$ & $91(6,4 \%)$ & $<0,001$ & $2,7(1,8-3,9)$ \\
\hline Pré-eclâmpsia & $35(11 \%)$ & $23(2 \%)$ & $<0,001$ & $7,3(4,3-12,6)$ \\
\hline Gemelaridade & $80(25 \%)$ & $40(3 \%)$ & $<0,001$ & $11,4(7,6-17,0)$ \\
\hline $\begin{array}{l}\text { Peso de nascimento (g) (média } \pm \text { DP) } \\
\quad<2500 \mathrm{~g} \\
\quad>4000 \mathrm{~g}\end{array}$ & $\begin{array}{c}2456 \pm 439 g \\
171(53 \%) \\
1(0,3 \%)\end{array}$ & $\begin{array}{c}3019 \pm 398 \\
130(9 \%) \\
12(1 \%)\end{array}$ & $\begin{array}{c}<\mathbf{0 , 0 0 1} 1^{\dagger} \\
<\mathbf{0 , 0 0 1} \\
0,56\end{array}$ & $11,2(8,4-14,8)$ \\
\hline Pequeno para a idade gestacional & $12(4 \%)$ & $20(1 \%)$ & 0,005 & $2,7(1,3-5,6)$ \\
\hline Grande para a idade gestacional & $12(4 \%)$ & $15(1 \%)$ & $<0,001$ & $3,6(1,7-7,8)$ \\
\hline Reanimação na sala de partos & $32(10 \%)$ & $75(5 \%)$ & 0,003 & $2(1,3-3,0)$ \\
\hline Parto espontâneo & $225(70 \%)$ & $991(70 \%)$ & 0,56 & \\
\hline $\begin{array}{l}\text { Tipo de parto } \\
\text { vaginal } \\
\text { cesariana }\end{array}$ & $\begin{array}{c}236(73 \%) \\
88(27 \%)\end{array}$ & $\begin{array}{c}1109(78 \%) \\
312(22 \%)\end{array}$ & 0,048 & $1,3(1,0-1,7)$ \\
\hline
\end{tabular}

* Qui-quadrado, exceto em +; † Teste t de Student; OR, odds ratio; IC, intervalo de confiança; DP, desvio padrão.

Dados apresentados em $\mathrm{n}(\%)$ exceto quando especificado.

Tabela 2. Comparação da morbidade e mortalidade neonatal entre os recém-nascidos pré-termo tardios (RNPT-T) e os recém-nascidos de termo precose (RNT-P). Amostra total: 1745 recém-nascidos. Maternidade Bissaya Barreto, Centro Hospitalar e Universitário de Coimbra, Portugal, 2014/2015.

\begin{tabular}{|c|c|c|c|c|}
\hline Morbimortalidade neonatal - n (\%) & $\begin{array}{c}\text { RN } 34^{0 / 7}-36^{6 / 7} \\
(n=324)\end{array}$ & $\begin{array}{c}\text { RN } 37^{0 / 7}-38^{6 / 7} \\
(n=1421)\end{array}$ & $\mathbf{p}^{*}$ & ORa $(95 \%$ IC) \\
\hline Internação em UCIN & $91(28 \%)$ & $84(6 \%)$ & $<0,001$ & $3,9(2,6-5,9)$ \\
\hline TTRN & $43(14 \%)$ & $33(2 \%)$ & $<0,001$ & $6,4(4,0-10,3)$ \\
\hline Necessidade de oxigênio & $18(6 \%)$ & $17(1 \%)$ & $<0,001$ & $5,5(2,4-12,5)$ \\
\hline Necessidade de ventilação & $3(1 \%)$ & $3(0,2 \%)$ & 0,082 & \\
\hline Hipoglicemia & $30(9 \%)$ & $29(2 \%)$ & 0,045 & $4,9(2,9-8,2)$ \\
\hline Dificuldades alimentares & $107(33 \%)$ & $99(7 \%)$ & $<0,001$ & $6,6(4,8-8,9)$ \\
\hline Hiperbilirrubinemia & $149(46 \%)$ & $215(15 \%)$ & $<0,001$ & $4,8(3,7-6,2)$ \\
\hline Sepsis & $5(2 \%)$ & $3(0,2 \%)$ & 0,024 & $4,4(1,2-15,4)$ \\
\hline Lesão do plexo braquial & $2(1 \%)$ & $3(0,2 \%)$ & 0,23 & \\
\hline Apneia & $5(2 \%)$ & $4(0,3 \%)$ & 0,014 & $5,5(1,4-20,1)$ \\
\hline Hemorragia peri/intraventricular & $4(1 \%)$ & $2(0,1 \%)$ & 0,02 & $8,8(1,6-48)$ \\
\hline Encefalopatia hipóxico-isquêmica & $1(0,3 \%)$ & $3(0,2 \%)$ & 0,56 & \\
\hline Reinternação & $4(1 \%)$ & $5(0,4 \%)$ & 1,0 & \\
\hline Óbito & $1(0,3 \%)$ & $1(0,07 \%)$ & 0,34 & \\
\hline Duração da internação (dias) - média \pm DP & $5,7 \pm 3,9$ & $3,1 \pm 1,4$ & $<0,001^{\dagger}$ & \\
\hline Internação $\geq 5$ dias & $152(47 \%)$ & $133(9 \%)$ & $<0,001$ & $8,6(6,6-11,3)$ \\
\hline Aleitamento materno exclusivo na alta & $238(73,5 \%)$ & $1325(93 \%)$ & $<0,001$ & $0,2(0,15-0,3)$ \\
\hline
\end{tabular}

* Qui-quadrado, exceto em †; † Teste T de Student; UCIN, unidade de cuidados intensivos neonatais; TTRN, taquipneia transitória do recém-nascido; ORa, odds ratio ajustada ao peso de nascimento, gemelaridade, patologia materna e tipo de parto; IC, intervalo de confiança; DP, desvio padrão.

Dados apresentados em $\mathrm{n}(\%)$ exceto quando especificado. 
Tabela 3. Morbidade/mortalidade neonatal de acordo com idade gestacional e odds ratio calculado em relação ao grupo de recém-nascidos de termo precoce (37-38 semanas: $n=1421)$. Amostra total: 1745 recém-nascidos. Maternidade Bissaya Barreto, Centro Hospitalar e Universitário de Coimbra, Portugal, 2014/2015.

\begin{tabular}{|c|c|c|c|c|c|}
\hline Variáveis & $\begin{array}{c}34 \mathrm{~S} \\
(\mathrm{n}=60)\end{array}$ & $\begin{array}{c}35 \mathrm{~S} \\
(n=102)\end{array}$ & $\begin{array}{c}36 \mathrm{~S} \\
(n=162)\end{array}$ & $\begin{array}{c}37 S \\
(n=400)\end{array}$ & $\begin{array}{c}38 \mathrm{~S} \\
(\mathrm{n}=1021)\end{array}$ \\
\hline Internação em UCIN & $\begin{array}{c}28(47 \%) \\
\text { OR-13,4 (7,7-23,3) }\end{array}$ & $\begin{array}{c}30(29 \%) \\
\text { OR-6,4 (4,0-10,3) }\end{array}$ & $\begin{array}{c}30(19 \% \\
\text { OR-3,5 (2,2-5,5) }\end{array}$ & $45(11 \%)$ & $42(4 \%)$ \\
\hline TTRN & $\begin{array}{c}13(22 \%) \\
\text { OR-11,6 (5,8-23,5) }\end{array}$ & $\begin{array}{c}17(17 \%) \\
\text { OR-8,4 (4,5-15,7) }\end{array}$ & $\begin{array}{c}13(8 \%) \\
\text { OR-3,7 (1,9-7,1) }\end{array}$ & $12(3 \%)$ & $21(2 \%)$ \\
\hline Hipoglicemia & $\begin{array}{c}11(18,3 \%) \\
\text { OR-10,8 (5,1-22,8) }\end{array}$ & $\begin{array}{c}11(10,8 \%) \\
\text { OR-5,8 (2,8-11,9) }\end{array}$ & $\begin{array}{c}8(4,9 \%) \\
\text { OR-2,5 (1,1-5,6) }\end{array}$ & $11(2,8 \%)$ & $18(1,8 \%)$ \\
\hline Hiperbilirrubinemia & $\begin{array}{c}35(58,3 \%) \\
\text { OR-7, } 9(\mathbf{4 , 6 - 1 3 , 4 )}\end{array}$ & $\begin{array}{c}54(52,9 \%) \\
\text { OR-6,3 (4,2-9,6) }\end{array}$ & $\begin{array}{c}60(37,0 \%) \\
\text { OR-3,3 (2,3-4,7) }\end{array}$ & $108(27,0 \%)$ & $107(10,5 \%)$ \\
\hline Reanimação & $\begin{array}{c}11(18,3 \%) \\
\text { OR-4,0 (2,0-8,1) }\end{array}$ & $\begin{array}{c}10(9,8 \%) \\
\text { OR-1,9 }(1,0-3,9)\end{array}$ & $\begin{array}{c}11(6,8 \%) \\
\text { OR-1,3 }(0,7-2,5)\end{array}$ & $26(6,5 \%)$ & $49(4,8 \%)$ \\
\hline Oxigenioterapia & $\begin{array}{c}7(11,7 \%) \\
\text { OR-10,9 }(\mathbf{4}, 3-27,4)\end{array}$ & $\begin{array}{c}7(6,9 \%) \\
\text { OR-6,1 (2,5-15,0) }\end{array}$ & $\begin{array}{c}5(3,1 \%) \\
\text { OR-2,6 }(0,9-7,2)\end{array}$ & $4(1,0 \%)$ & $13(1,3 \%)$ \\
\hline Necessidade de ventilação & $\begin{array}{c}2(3,3 \%) \\
\text { OR-16,3 (2,7-99) }\end{array}$ & $\begin{array}{c}1(1,0 \%) \\
\text { OR-6,1 }(2,5-15)\end{array}$ & $\begin{array}{c}0(0,0 \%) \\
\text { OR-0,9 }(0,9-1,0)\end{array}$ & $0(0,0 \%)$ & $3(0,3 \%)$ \\
\hline Dificuldades alimentares & $\begin{array}{c}37(61,7 \%) \\
\text { OR-21,5 (12,3-37,6) }\end{array}$ & $\begin{array}{c}43(42,2 \%) \\
\text { OR-9,7 (6,3-15,2) }\end{array}$ & $\begin{array}{c}27(16,7 \%) \\
\text { OR-2,7 (1,7-4,2) }\end{array}$ & $45(11,3 \%)$ & $54(5,3 \%)$ \\
\hline Sepsis & $\begin{array}{c}1(1,7 \%) \\
\text { OR-4,8 }(0,6-41,7)\end{array}$ & $\begin{array}{c}1(1,0 \%) \\
\text { OR-2,8 }(0,3-24,2)\end{array}$ & $\begin{array}{c}3(1,9 \%) \\
\text { OR-5,3 }(\mathbf{1}, \mathbf{3 - 2 2 , 6 )}\end{array}$ & $2(0,5 \%)$ & $3(0,3 \%)$ \\
\hline Lesão do plexo braquial & $\begin{array}{c}0(0,0 \%) \\
\text { OR-0,9 }(0,9-1,0)\end{array}$ & $\begin{array}{c}1(1,0 \%) \\
\text { OR-4,7 }(0,5-46)\end{array}$ & $\begin{array}{c}1(0,6 \%) \\
\text { OR-2,9 }(0,3-28)\end{array}$ & $0(0,0 \%)$ & $3(0,3 \%)$ \\
\hline Apneia & $\begin{array}{c}3(5,0 \%) \\
\text { OR-26,8 (4,4-164) }\end{array}$ & $\begin{array}{c}2(2,0 \%) \\
\text { OR-7, } 1(\mathbf{1}, \mathbf{3}-\mathbf{3 9}, \mathbf{1})\end{array}$ & $\begin{array}{c}0(0,0 \%) \\
\text { OR-0,9 }(0,9-1,0)\end{array}$ & $2(0,5 \%)$ & $2(0,2 \%)$ \\
\hline HIPV & $\begin{array}{c}3(5,0 \%) \\
\text { OR-53,7 (5,5-524) }\end{array}$ & $\begin{array}{c}1(1,0 \% \\
\text { OR-7, } 0(0,6-78)\end{array}$ & $\begin{array}{c}0(0,0 \%) \\
\text { OR-0,9 }(0,9-1,0)\end{array}$ & $1(0,3 \%)$ & $1(0,1 \%)$ \\
\hline Reinternação & $\begin{array}{c}2(3,3 \%) \\
\text { OR-3,5 }(0,8-15,6)\end{array}$ & $\begin{array}{c}2(2,0 \%) \\
\text { OR-2,0 (0,5-9) }\end{array}$ & $\begin{array}{c}0(0,0 \%) \\
\text { OR-0, } 9(0,9-1,0)\end{array}$ & $7(1,8 \%)$ & $7(0,7 \%)$ \\
\hline Perda ponderal > $10 \%$ & $\begin{array}{c}5(8,3 \%) \\
\text { OR-1, } 4(0,6-3,6)\end{array}$ & $\begin{array}{c}8(7,8 \%) \\
\text { OR-1,3 }(0,6-2,8)\end{array}$ & $\begin{array}{c}7(4,3 \%) \\
\text { OR-0,7 }(0,3-1,5)\end{array}$ & $26(6,5 \%)$ & $60(5,9 \%)$ \\
\hline Internamento $\geq 5$ dias & $\begin{array}{c}48(80,0 \%) \\
\text { OR-38,7 (20,1-74,7) }\end{array}$ & $\begin{array}{c}55(53,9 \%) \\
\text { OR-11,3 (7,3-17,3) }\end{array}$ & $\begin{array}{c}48(29,6 \%) \\
\text { OR- } \mathbf{4 , 1}(\mathbf{2}, \mathbf{8}-\mathbf{6}, \mathbf{0})\end{array}$ & $67(16,8 \%)$ & $66(6,5 \%)$ \\
\hline LM exclusivo na alta & $\begin{array}{c}19(31,7 \%) \\
\text { OR-0,03 (0,02-0,06) }\end{array}$ & $\begin{array}{c}74(72,5 \%) \\
\text { OR-0,2 (0,1-0,3) }\end{array}$ & $\begin{array}{c}145(89,5 \%) \\
\text { OR-0,6 }(0,4-1,0)\end{array}$ & $368(92,0 \%)$ & $957(93,7 \%)$ \\
\hline Óbito & $\begin{array}{c}0(0,0 \%) \\
\text { OR-0, } 9(0,9-1,0)\end{array}$ & $\begin{array}{c}0(0,0 \%) \\
\text { OR-0, } 9(0,9-1,0)\end{array}$ & $\begin{array}{c}1(0,6 \%) \\
\text { OR-8,8 (0,5-14,1) }\end{array}$ & $0(0,0 \%)$ & $1(0,1 \%)$ \\
\hline Duração média da internação $\pm D P$ & $9,0( \pm 5,0)$ & $6,0( \pm 3,8)$ & $4,2( \pm 2,6)$ & $3,5( \pm 1,8)$ & $3,0( \pm 1,2)$ \\
\hline
\end{tabular}

S, semanas de idade gestacional; UCIN, unidade de cuidados intensivos neonatais; TTRN, taquipneia transitória do recém-nascido; HPIV, hemorragia peri-intraventricular; EHI, encefalopatia hipóxico-isquêmica; SAM, síndrome de aspiração meconial; LM, leite materno; DP, desvio padrão.

Dados apresentados como odds ratio (intervalo de confiança 95\%). Em negrito os resultados estatisticamente significativos.

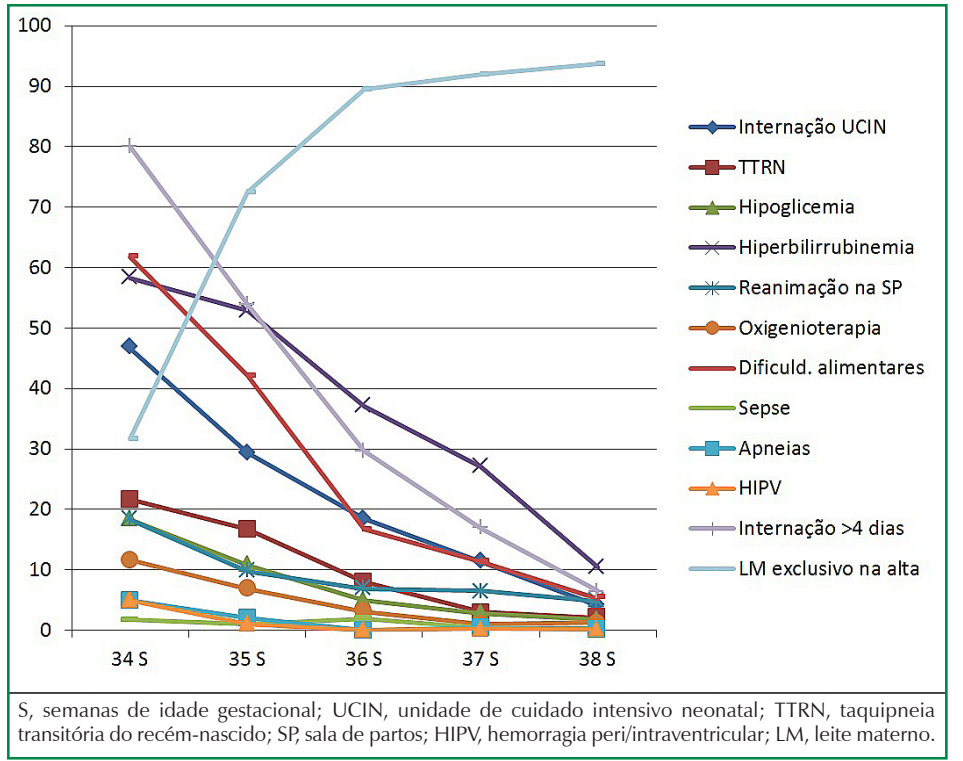

Figura 1. Morbidade neonatal por idade gestacional (valores referentes à Tabela 3).

Amostra total: 1745 recém-nascidos.

Maternidade Bissaya Barreto, Centro Hospitalar e Universitário de Coimbra, Portugal, 2014/2015. 


\section{DISCUSSÃO}

Este estudo demonstra a maior morbidade neonatal associada aos RNPT-T em comparação com os RNT-P, apesar da pouca diferença entre semanas de gestação. As causas para isso têm a ver com a imaturidade fisiológica e metabólica em função da menor idade gestacional $[2,18]$.

Identificamos um risco seis vezes maior de TTRN e cinco vezes maior de necessidade de oxigenioterapia. Os distúrbios respiratórios são dos mais frequentes nos RNPT-T, pela imaturidade pulmonar, a qual implica em estrutura e vascularização alveolar que levam a dificuldade na reabsorção do fluido alveolar, menor produção de surfactante e consequente diminuição das trocas gasosas [19].

O maior risco de apneias, que também foi encontrado neste estudo, pode ser devido quer à imaturidade do sistema nervoso central, ligada ao maior risco de apneias centrais, quer à imaturidade dos sistemas metabólicos de resposta à hipercapnia e hipoxia, e ao menor tônus dos músculos dilatadores da via aérea superior [2].

Houve maior necessidade de reanimação na sala de partos para os RNPT-T, o que pode ser explicado por vários fatores. Por um lado, a patologia materna, também associada a uma menor idade gestacional dos recém-nascidos, por outro a imaturidade destes, que diminui as respostas fisiológicas compensatórias à adaptação ao ambiente extrauterino [2,20].

O maior risco de hipoglicemia nos RNPT-T pode estar relacionado à porcentagem significativa de história de diabetes gestacional nesse grupo, mas também às dificuldades alimentares, com diminuição do aporte enteral necessário, e/ou pela deficiente resposta metabólica à diminuição abrupta da glicose materna inerente à imaturidade dos mecanismos de glicogenólise e neoglicogênese [21].

A hiperbilirrubinemia nos RNPT-T é mais elevada e prolongada devido à imaturidade da função hepática, com diminuição da conjugação e eliminação da bilirrubina, e às dificuldades alimentares nesse grupo de recém-nascidos. Deve ser levado em conta também que os critérios para fototerapia utilizados para idades gestacionais menores são mais baixos, pelo risco de neurotoxicidade e encefalopatia relacionadas à maior permeabilidade da barreira hemato-encefálica e menor eficácia dos mecanismos de proteção neuronal [22].

As dificuldades alimentares foram registradas numa percentagem significativamente maior nos RNPT-T e com menor percentagem de aleitamento materno exclusivo na alta. A menor taxa de aleitamento materno na alta traz desvantagens sobejamente conhecidas quer para o recém-nascido quer para a mãe. A menor taxa de aleitamento materno exclusivo nos RNPT-T pode estar relacionada, entre outros fatores, aos reflexos oromotores ainda pouco desenvolvidos e a menores períodos de vigília em relação aos RNT-P. [19] As dificuldades alimentares podem aumentar os riscos de hipoglicemia, desidratação e hiperbilirrubinemia, repercutindo de forma importante na maior duração da hospitalização desses recém-nascidos.

A duração da hospitalização foi significativamente maior nos RNPT-T. A maior incidência de complicações no período neonatal atrasa a alta para o domicílio, por vezes com necessidade de internamento em UCIN. Isto acarreta um aumento de recursos necessários para o cuidado destes $\mathrm{RN}$ e o consequente aumento de custos [2].

Apesar do pouco número de casos de sepsis, o risco dessa intercorrência foi mais de quatro vezes maior entre os casos. O parto prematuro pode ser desencadeado por infeção materna, e por outro lado, o recém-nascido pré-termo apresenta imaturidade do sistema imunitário e é submetido a maior número de procedimentos durante a hospitalização.

O sistema nervoso central nos RNPT-T encontrase ainda numa fase de desenvolvimento e vulnerável a qualquer insulto na vida extrauterina [19]. Assim, há o risco no período neonatal de complicações como a HPIV, que neste estudo, apesar dos poucos casos, foi quase nove vezes maior em relação aos RNT-P.

A imaturidade fisiológica dos recém-nascidos prétermo em geral não é a única explicação para o aumento de morbidade neonatal nos RNPT-P. As causas dessa prematuridade, a patologia materna e placentária e a exposição a fatores adversos in utero devem ser levadas em conta [20]. Neste estudo houve uma associação significativa entre RNPT-T e patologias relacionadas com a gestação, tais como diabetes gestacional, hipertensão arterial e pré-eclâmpsia. Houve também uma associação maior entre RNPT-T e a gemelaridade. $\mathrm{O}$ fato de haver uma maior percentagem de recémnascidos PIG pode ser também indicativo de fatores de estresse pré-natais responsáveis pela RCIU e pela prematuridade [20].

Os resultados deste estudo vão de encontro ao referido na literatura, que demonstra a maior morbidade dos RNPT-T no período neonatal e também a longo prazo $[9,10,18,23-25]$. Apesar de muitas vezes serem encarados como recém-nascidos de termo por terem peso de nascimento semelhante, a imaturidade fisiológica e metabólica dos RNPT-T leva a esses riscos maiores. A diferença de idade gestacional para 
os RNT-P, mesmo que pequena, é significativa em termos do maior número de complicações. Esse fato causa impacto na organização dos cuidados perinatais, sobretudo quando há elevada prevalência de partos prematuros $[24,25]$.

Assim, a maior morbidade dos RNPT-T e o maior risco de complicações requerem um seguimento médico adequado e diferenciado no período neonatal e posteriormente, pelas complicações que poderão advir da prematuridade. Se para gestações abaixo das 34 semanas essa abordagem é consensual, para idade gestacional entre as $34^{0 / 7}$ e as $36^{6 / 7}$ semanas completas de gestação o maior risco de complicações deve ser cada vez mais valorizado e tido em conta na prática clínica.

\section{NOTAS}

Publicação prévia

Estudo apresentado no $17^{\circ}$ Congresso Nacional de Pediatria de Portugal, em novembro de 2016. O resumo foi publicado no suplemento da Acta Pediátrica Portuguesa dedicado aos anais do congresso: Acta Pediátrica Portuguesa, volume 47, Supl (2016), disponível em http://actapediatrica.spp.pt/article/view/10241/7402.

\section{Apoio financeiro}

Este estudo não recebeu apoio financeiro de fontes externas.

Declaração de conflito de interesses

Os autores declaram não haver conflitos de interesses relevantes ao conteúdo deste estudo, informam ter tido acesso a todos os dados obtidos e assumem completa responsabilidade pela integridade dos resultados.

\section{REFERÊNCIAS}

1. World Health Organization. ICD-10: International statistical classification of diseases and related health problems, 10th revision. Geneva: WHO; 2004.

2. Engle WA, Tomashek KM, Wallman C. "Late-Preterm" Infants: A Population at Risk. Pediatrics. 2007;120(6):1390-401. https://doi.org/10.1542/peds.2007-2952

3. Barros J, Clode N, Graca L. Prevalence of Late Preterm and Early Term Birth in Portugal. Acta Med Port. 2016;29(4): 249-53. https://doi.org/10.20344/amp.6523

4. Raju TNK, Higgins RD, Stark AR, Leveno KJ. Optimizing Care and Outcome for Late-Preterm (Near-Term) Infants: A Summary of the Workshop Sponsored by the National Institute of Child Health and Human Development. Pediatrics. 2006;118(3):1207-14. https://doi.org/10.1542/peds.2006-0018

5. American College of Obstetricians and Gynecologists. Definition of term pregnancy. Committee Opinion N 579 . Obstet Gynecol. 2013(122):1139-40. https://doi.org/10.1097/01.AOG.0000437385.88715.4a

6. Tita ATN, Landon MB, Spong CY, Lai Y, Leveno KJ, Varner MW, et al. Timing of Elective Repeat Cesarean Delivery at Term and Neonatal Outcomes. N Engl J Med. 2009;360(2):111-20. https://doi.org/10.1056/NEJMoa0803267

7. Engle WA. Morbidity and mortality in late preterm and early term newborns: A continuum. Clin Perinatol. 2011;38(3): 493-516. https://doi.org/10.1016/j.clp.2011.06.009

8. Escobar GJ, Clark RH, Greene JD. Short-Term Outcomes of Infants Born at 35 and 36 Weeks Gestation: We Need to Ask More Questions. Seminars in Perinatology. 2006;30(1):28-33. https://doi.org/10.1053/j.semperi.2006.01.005

9. Machado Júnior LC, Passini Júnior R, Rodrigues Machado Rosa I. Late prematurity: a systematic review. J Pediatr (Rio J). 2014;90(3):221-31. https://doi.org/10.1016/j.jped.2013.08.012

10. Teune MJ, Bakhuizen S, Gyamfi Bannerman C, Opmeer BC, van Kaam AH, van Wassenaer AG, Morris JM, Mol BW. A systematic review of severe morbidity in infants born late preterm. Am J Obstet Gynecol. 2011;205(4):374.e1-.e9. https://doi.org/10.1016/j.ajog.2011.07.015

11. Gkentzi D, Dimitriou G. Long-Term Outcome of Infants Born Late Preterm. Curr Pediatr Rev. 2014;10(4):263-7. https:// doi.org/10.2174/1573400510666141114224257

12. Direção Geral de Saúde. Programa de vigilância epidemiológica da infeção nosocomial em unidades de cuidados intensivos neonatais [Internet]. Lisboa: DGS; 2007 [cited November 2016]. Available from: https://www.dgs.pt/programanacional-de-controlo-da-infeccao/ficheiros-de-upload/ucin-protocolo-pdf.aspx

13. Executive summary: Neonatal encephalopathy and neurologic outcome, second edition. Report of the American College of Obstetricians and Gynecologists' Task Force on Neonatal Encephalopathy. Obstet Gynecol. 2014;123(4):896-901. https://doi.org/10.1097/01.AOG.0000445580.65983.d2

14. Okumura A, Kidokoro H, Shoji H, Nakazawa T, Mimaki M, Fujii K, et al. Kernicterus in preterm infants. Pediatrics. 2009;123(6):e1052-8. https://doi.org/10.1542/peds.2008-2791

15. National Collaborating Centre for Women's and Children's Health. NICE Clinical Guidelines, No. 98: Neonatal Jaundice [Internet]. London: RCOG Press; 2010 [cited November 2016]. Available from: http://www.ncbi.nlm.nih.gov/books/ NBK65119

15. Volpe JJ. Intracranial hemorrhage: germinal matrix-intraventricular hemorrhage of the premature infant. In: JJ Volpe, editors. Neurology of the newborn. 5th ed. Philadelphia: Saunders/Elsevier; 2008. p 517-588.

17. Fenton TR. A new growth chart for preterm babies: Babson and Benda's chart updated with recent data and a new format. BMC Pediatr. 2003;3:13. https://doi.org/10.1186/1471-2431-3-13 
18. Dong Y, Yu J-L. An overview of morbidity, mortality and long-term outcome of late preterm birth. World J Pediatr. 2011;7(3):199-204. https://doi.org/10.1007/s12519-011-0290-8

19. Horgan MJ. Management of the Late Preterm Infant: Not Quite Ready for Prime Time. Pediatr Clin North Am. 2015;62(2):439-51. https://doi.org/10.1016/j.pcl.2014.11.007

20. Brown HK, Speechley KN, Macnab J, Natale R, Campbell MK. Neonatal morbidity associated with late preterm and early term birth: the roles of gestational age and biological determinants of preterm birth. Int J Epidemiol. 2014;43(3):802-14. https://doi.org/10.1093/ije/dyt251

21. Ward M, Deshpande S. Metabolic adaptation at birth. Semin Fetal Neonatal Med. 2005;10(4):341-50. https://doi. org/10.1016/j.siny.2005.04.001

22. Sarici SÜ, Serdar MA, Korkmaz A, Erdem G, Oran O, Tekinalp G, et al. Incidence, Course, and Prediction of Hyperbilirubinemia in Near-Term and Term Newborns. Pediatrics. 2004;113(4):775-80. https://doi.org/10.1542/ peds.113.4.775

23. Chan E, Leong P, Malouf R, Quigley MA. Long-term cognitive and school outcomes of late-preterm and early-term births: a systematic review. Child Care Health Dev. 2016;42(3):297-312. https://doi.org/10.1111/cch.12320

24. Gill J, Boyle E. Outcomes of infants born near term. Arch Dis Child 2017;102:194-8. https://doi.org/10.1136/ archdischild-2015-309584

25. Boyle EM, Johnson S, Manktelow B, et al. Neonatal outcomes and delivery of care for infants born late preterm or moderately preterm: a prospective population-based study. Archives of Disease in Childhood Fetal and Neonatal Edition. 2015;100(6):F479-F485. https://doi.org/10.1136/archdischild-2014-307347 